\title{
Treatment of Opioid-induced Constipation: A Therapeutic Update
}

\section{Martin M Zdanowicz*}

University of Miami School of Nursing and Health Studies, 5030 Brunson Drive, Coral Gables, Florida, USA

\begin{abstract}
Opioids remain the most effective therapeutic agents for the management of moderate to severe pain. A significant number of patients receive long-term opioid therapy for chronic pain. While a number of opioid side effects such as sedation, nausea and vomiting, and respiratory depression diminish over time due to the development of tolerance, the constipating effects of opioid persist throughout the course of therapy. Opioid-induced constipation (OIC) is associated with marked bowel dysfunction that can significantly impact a patient's quality of life and affect their drug adherence. A step-wise approach to treating OIC should be considered which includes life style modifications, administration of laxatives and stool-softeners, and if necessary, pharmacologic agents to enhance bowel motility and antagonize peripheral $\mu$-opioid receptors. Although OIC is a significant adverse effect associated with opioid use, clinical studies have shown that a number of effective treatment strategies are available for both preventing OIC or reducing its severity.
\end{abstract}

Keywords: Opioids; Opioid-induced constipation; $\mu$-opioid receptors; Laxatives

\section{Introduction}

In 2013, an estimated 207 million opioid prescriptions were dispensed by retail pharmacies in the U.S. (https://www.drugabuse. gov/about-nida/legislative-activities/testimony-to-congress/2016/ americas-addiction-to-opioids-heroin-prescription-drug-abuse). While opioids are highly efficacious for the treatment of acute and chronic pain, they may cause a number of significant side effects. One of the most commonly reported side effects of opioid use is opioidinduced constipation (OIC). According to recent studies, $41-47 \%$ of patients using opioids for chronic pain experience significant OIC [1,2]. In patients on chronic opioid therapy, the degree of OIC can be quite severe and even debilitating [3]. A number of therapeutic interventions are currently available to treat OIC with varying degrees of efficacy. Treatment for OIC may include over-the-counter (OTC) options such as stimulant laxatives, stool softeners and osmotic laxatives, as well as prescription drugs that antagonize peripheral $\mu$-opioid receptors, and directly stimulate intestinal secretions and motility. The various treatment options will be discussed below with emphasis on their mechanism of action, efficacy, and potential side-effects.

\section{Opioids and OIC}

The human opioid system is highly complex and includes three main endogenous opioid receptors ( $\mu, \kappa$ and $\delta$ receptors), as well as a number of endogenous opioid peptides such as endorphins, enkephalins, and dynorphins which activate these receptors. Opioid receptors are widely distributed in the brain and spinal cord, as well as in a variety of peripheral tissues such as the gut, airways, blood vessels, and heart. When activated, the opioid receptors located in the brain and spinal cord mediate the analgesic effects of the opioids. Unfortunately, activation of opioid receptors in the gut can significantly impair intestinal activity and lead to OIC.

$\mathrm{Mu}$-opioid receptors, and to a lesser extent $\kappa$-opioid receptors, are expressed extensively throughout the gastrointestinal tract and enteric nervous system. Activation of these opioid receptors decreases peristalsis, inhibits intestinal transit, increases intestinal fluid absorption and decreases intestinal secretions. All of these effects combine to cause significant constipation [4]. A clinical syndrome of opioid-induced bowel dysfunction may occur with chronic opioid use that is characterized by abdominal pain, hard stools, fecal impaction, anorexia, nausea and vomiting [5].

The presence of OIC can significantly impact a patient's quality of life and can lead to dose reduction or even cessation of opioid pain therapy. A 2009 study by Bell et al. [6], examined the prevalence, severity and impact of OIC in 322 patients taking daily opioids. The investigators found that $81 \%$ of the patients in their study reported OIC and that the majority of patients with OIC reported at least a moderate negative impact on quality of life and activities of daily living. One third of patients with OIC missed doses, decreased dosage or stopped using opioids completely in order to improve their bowel function. A second study by Bell [7] found that patients with OIC were more likely to take time off from work and were less productive in both their work and home environments.

A 2010 study by Hjalte, et al. [8], examined the direct and indirect costs of OIC in a population $(n=331)$ of patients treated with opioids for six months. The investigators reported that the total cost per patient per month was significantly higher in those patients who experienced significant OIC when compared to those who had mild, moderate or no symptoms of OIC. The costs examined included the direct cost of inpatient needs, out-patient visits, supplemental drug costs and indirect costs related to sick leave and lost productivity at the workplace. Patients in this study with more severe OIC also reported significantly lower quality life scores than those with mild or no symptoms. Data also suggests that health care providers may not always be aware of the fact that patients are experiencing significant OIC. In a recent study with 489 patients receiving opioid therapy, $94 \%$ of patients reported suffering from constipation; however, only $61 \%$ of their healthcare providers knew that a patient in their care was suffering from significant constipation [9].

*Corresponding author: Martin M Zdanowicz, Associate Dean for Health Studies University of Miami School of Nursing and Health Studies, 5030 Brunson Drive, Coral Gables, Florida-33124, USA, Tel: 3052844680; E-mail: mzdanowicz@miami.edu Received May 06, 2016; Accepted June 07, 2016; Published June 14, 2016

Citation: Zdanowicz MM (2016) Treatment of Opioid-induced Constipation: A Therapeutic Update. Adv Practice Nurs 2:118. doi:10.4172/2573-0347.1000118

Copyright: $\odot 2016$ Zdanowicz MM. This is an open-access article distributed under the terms of the Creative Commons Attribution License, which permits unrestricted use, distribution, and reproduction in any medium, provided the original author and source are credited. 


\section{Opioid Tolerance and OIC}

With continued opioid use, patients often exhibit tolerance to the analgesic effects of the opioids. The mechanism of opioid tolerance is likely related to down-regulation (decreased numbers) or desensitization of $\mu$-opioid receptors in the CNS. Binding of opioids to the $\mu$ receptor activates numerous downstream intracellular pathways. Activated G-protein-dependent signaling pathways appear involved in receptor desensitization while G-protein-independent signaling pathways appear to facilitate $\mu$ receptor endocytosis and subsequent downregulation of receptors [10]. Fortunately, tolerance to the respiratory depressant effects of the opioids also develops in parallel to the tolerance seen with the analgesic effects. Interestingly however, tolerance does not occur to the constipating effect of the opioids. A recent comprehensive review by Akbarali [11] reported that tolerance to the inhibitory effects of opioids does appear to occur in the upper GI tract, but tolerance does not develop in the colon and rectum. This regional difference in the development of GI tolerance appears to be related to differences in signaling and regulation of opioid receptors in the two anatomical regions. Specifically, the activity of the regulatory protein $\beta$-arrestin 2 may differ between the small and large intestines. In animal studies, chronic administration of morphine to the ileum resulted in down-regulation of $\beta$-arrestin 2 and the development of tolerance while chronic administration of morphine in colon does not affect $\beta$-arrestin2 levels and as a result tolerance does not occur [11]. An understanding of the regional differences in $\mu$-opioid receptor regulation is important because it not only enhances our overall understanding of the molecular basis for opioid tolerance but may also lead to the identification of possible future therapeutic targets for the treatment of OIC.

\section{Diagnosis of OIC}

Opioid-induced constipation and opioid bowel dysfunction presents with a myriad of symptoms. The Rome III criterion for functional gastrointestinal disorders presents detailed diagnostic criteria for constipation. Diagnosis of OIC should begin with a detailed patient history that includes frequency of bowel movements, the consistency of stool, and the presence of straining, pain, nausea and vomiting. A physical examination should also be conducted that includes bowel sounds, and abdominal palpation for firmness, distention and the presence of pain. The possibility of fecal impaction should also be assessed in patients with persistent and severe constipation. Health care providers should also be aware of the possibility that the constipation observed in their patients might be related to an ongoing disease processes. In cancer patients for example, tumors in the intestine or abdomen may cause bowel obstruction. Likewise, tumors developing in or around the spine may compress nerves that innervate the gastrointestinal tract and thus impair gastric motility and function. A number of other drugs that a patient might be taking such as anticholinergics, tricyclic antidepressants, antihistamines, calcium or iron supplements and aluminum-containing antacids might also be contributing to their constipation.

The symptoms of opioid-induced constipation and bowel dysfunction:

- Constipation - 3 or fewer bowel movements per week

- Abdominal cramping, bloating and distention

- Dry, hard stools

- Pain, straining
- Incomplete evacuation

- Delayed digestions

- Nausea, vomiting

Rome III functional constipation diagnostic criteria ${ }^{\star}$

- Must include two or more of the following:

- Straining during at least $25 \%$ of defecations

- Lumpy or hard stools in at least $25 \%$ of defecations

- Sensation of incomplete evacuation for at least $25 \%$ of defecations

- Sensation of anorectal obstruction/blockage for at least $25 \%$ of defecations

- Manual maneuvers to facilitate at least $25 \%$ of defecations (e.g., digital evacuation, support of the pelvic floor)

- Fewer than three defecations per week

- Loose stools are rarely present without the use of laxatives

- Insufficient criteria for irritable bowel syndrome

Criteria fulfilled for the last 3 months with symptom onset at least 6 months prior to diagnosis

\section{Treatment strategies for OIC}

A number of non-pharmacologic and pharmacologic options are available for the treatment and prevention of OIC. Although current treatment recommendations support the prophylactic use of various bowel regimens in patients receiving opioid therapy, definitive studies showing the superiority of one treatment regimen over another are currently lacking.

Non-pharmacologic management of OIC: Non-pharmacologic treatment options for the prevention and treatment of OIC should be a component of the overall strategy to prevent OIC and should start with the onset of opioid therapy. Non-pharmacologic interventions include adequate fluid and fiber intake, and increased physical activity. A study by Dukas, showed that physical activity two to six times per week was associated with a 35 percent lower risk of constipation in older women [12]. Abdominal massage has also been shown to help relieve constipation and improve patient quality of life without side effects. A recent study by Turan \& Asti, showed that patients with post-operative constipation who received bowel massage by nurses had reduced symptoms of constipation, increased stool output and improved quality of life with no significant adverse side effects [13]. However, a 2009 study by Lamas, et al. showed that while abdominal massage was useful for decreasing the severity of constipation symptoms, it did not lead to a decreased use of laxative. This finding suggests that abdominal massage may be used to complement the actions of laxatives but may not fully replace them [14]. Bowel "diaries" which track the frequency of bowel movements might also be helpful for determining the severity of the OIC that is occurring. While helpful, non-pharmacologic interventions are seldom successful alone for the management of OIC $[4,15]$.

OTC management of OIC: Pharmacologic interventions for OIC include both OTC agents as well as prescription drugs. Common OTC agents used to treat OIC include Stool softeners, bowel stimulants and osmotic laxatives. Stool softeners such as docusate sodium are essentially surfactants that facilitate the mixing of fats and water in the stool and are most useful in patients with hard, dry feces. Stool softeners alone have been shown to be of little benefit in patients with significant 
constipation [16] and are thus often combined with a stimulant laxatives for increased effectiveness. Stimulant laxatives such as senna and cascara directly stimulate the myenteric plexus in the gut which in turn enhances intestinal secretions and peristalsis. Although effective in OIC, there is evidence that certain patients may become tolerant to the therapeutic actions of stimulant laxatives over time [17]. Osmotic laxatives (polyethylene glycol (PEG), lactulose, magnesium hydroxide) may also be of value in treating OIC since they act by drawing water into the intestinal lumen which in turn increases intraluminal pressure and stimulates peristalsis. Magnesium-containing osmotic laxatives should be avoided in patients with renal insufficiency since they may result in hypermagnesemia. A comprehensive review of the literature by Ramkumar and Rao [18] found that while PEG and lactulose were both effective in treating chronic constipation, PEG was somewhat more effective than lactulose and had fewer overall side effects.

Patients with OIC should be counseled to avoid bulk laxatives that contain psyllium. Bulk laxatives are designed to draw fluid into the intestinal lumen where the indigestible fibers swell to form a bulky mass that stimulates peristalsis. However, given the fact that opioids impair peristalsis, the increased bulk is now more likely to become an immobile mass that can lead to bowel distention, pain and obstruction.

Although laxatives are generally well-tolerated, they can cause significant side effects such as diarrhea, abdominal pain, distention, nausea and vomiting. Likewise, laxatives do not directly affect or alter the mechanism by which opioids cause constipation. Despite their widespread use in OIC, Pappagallo [19] reported that only $46 \%$ of patients receiving laxative therapy for OIC achieved the desired symptomatic relief at least $50 \%$ of the time.

Prescription drugs for treating OTC: Lubiprostone (Amitiza) is an orally active prostone that selectively activates chloride channels in the intestine to increase both fluid secretion and intestinal motility. It is currently approved by the Food and Drug Administration (FDA) for the treatment of idiopathic chronic constipation, irritable bowel syndrome in women, and in adults with OIC being treated for chronic non-caner pain. A recent study by Jamal, et al. [20], examined the therapeutic effects of lubiprostone administration ( $24 \mu \mathrm{g}$ twice daily) in 431 adults with OIC who were being treated for chronic non-cancer pain. Administration of lubiprostone significantly improved spontaneous bowel movements, reduced straining and improved the consistency of stools. The percentage of patients who required "rescue" medications to treat their OIC was also significantly lower in the lubiprostone-treated group than the placebo-treated group. Lubiprostone appeared to be welltolerated by patients with the most common side effects being diarrhea, nausea, vomiting and abdominal pain. No serious adverse effects were seen in the lubiprostone-treated group. An earlier multi-center study by Cryer et al., also demonstrated that lubiprostone ( $24 \mu \mathrm{g}$ twice daily) significantly relieved symptoms of OIC for an 11 week period. The drug was well-tolerated and overall side effects were minimal [21].

Peripheral opioid receptor antagonists are agents that are designed to antagonize the effects of opioid at their receptors in the gut and periphery but not in the CNS. Using this strategy, peripheral opioid receptor antagonists counter the constipating effects of opioids that are mediated via peripheral opioid receptors but do not antagonize the analgesic effect of opioids that is mediated via central opioid receptors. Oral naloxone was tested initially for the treatment of OIC since it has a very limited systemic absorption when administered orally [22]. However, a lack of consistent therapeutic effects, difficulty with appropriate dosing and potential for antagonism of CNS opioid receptors significantly limits the use of naloxone in the treatment of
OIC. Later agents such are methylnaltrexone (Reistor), are quarternary $\mathrm{N}$-methyl derivatives of naloxone that are designed to have very low systemic bioavailability and very low CNS penetration, even if administered parenterally. Methylnaltrexone significantly improved symptoms of OIC without inducing opioid withdrawal or impairing analgesia $[23,24]$. The most common adverse effects associated with the use of methyltrexone were abdominal pain, flatulence and nausea. Serious adverse effects were not observed. Relistor was approved by the FDA in 2014 for the treatment of OIC. The normal dose of Relistor is 12 $\mathrm{mg} / 0.6 \mathrm{ml}$ s.c. once every other day but not more than once in a 24 hour period. Methylnaltrexone is contraindicated in patients with known or suspected bowel obstruction.

In 2014, the FDA also approved the drug naloxegol (Movantik) which is a pegylated (with added polyethylene glycol) form of naloxone. Naloxegol is the first orally administered, once a day, peripheral-acting mu opioid receptor antagonist (PAMORA) approved for treating OIC. A comprehensive review of studies related to the therapeutic efficacy of naloxegol was performed by Leonard \& Baker [25]. Their findings showed that naloxegol was effective for the long-term (9-12 weeks) of OIC. Significant improvements in spontaneous bowel movements were reported in several moderate to large scale clinical trials with patients experiencing OIC. The long-term (52 week) safety of naloxegol was also demonstrated. The most common side effects observed in patients receiving naloxegol were abdominal pain, nausea, diarrhea, and flatulence. Naloxegol is available in 12 or $25 \mathrm{mg}$ tablets for once a day dosing. A new high affinity, high potency PAMORA, Axelopran is also currently under development by Theravance Biopharma.

While opioid analgesics all exert their constipating effects through activation of opioid receptors, not all opioids agonists appear to be equally constipating. Thus an "opioid rotation" might be considered in selected patients to reduce the severity of constipation. A number of studies have reported that transdermal fentanyl was not only effective for the treatment of chronic cancer and non-cancer pain, but was also significantly better than oral morphine at preserving normal bowel function [26-28]. Despite these finding, a systematic review conducted by Dale [29] concluded that there was only a low level of evidence that opioid rotation significantly reduced the adverse effects of opioids.

Tapentadol (Nucynta), a dual-acting $\mu$-opioid receptor agonist and norepinephrine reuptake inhibitor, is another agent that might be considered for use in patients with chronic pain in place of a traditional opioid. Given that tapentadol has only moderate affinity for $\mu$-opioid receptors, one would anticipate significantly less opioidrelated adverse effects. In 2013 study by Kwong, et al. [30], patients with lower back pain or osteoarthritis who were treated with tapentadol had significantly better bowel function and less constipation that similar patients treated with oxycodone. Tramadol (Ultram) is a second dual action opioid analgesic that might be considered for use in selected patients. The affinity of tramadol for $\mu$-opioid receptors is less than that of tapentadol so it is less likely to cause significant OIC when compared to a traditional opioid or tapentadol [31].

Role of the advanced practice nurse in OIC: Advance practice nurses can play an important role in identifying and treating OIC since they are in regular contact with patients. They are also ideally situated with regards to identifying patients at high risk for OIC and ruling out other causes of chronic constipation. A detailed patient history should be obtained that includes diet, physical activity, and a review of all medications the patient is currently taking. A thorough patient examination should be conducted and accompanying signs and symptoms such nausea, vomiting or abdominal pain/distention should 
be noted. Nurses should monitor patient bowel habits as well as the quantity and quality of stools.

Advance practice nurses can also take a lead role in patient education to ensure that lifestyle changes such as increased fluid intake and physical activity are implemented. Nurses can also make certain that laxatives are prescribed prophylactically in patients they identify as high risk for OIC. Nurses can also play an important role in ensuring patient compliance with treatments designed to alleviate OIC as well as monitoring the effectiveness of such therapies once they are instituted.

\section{Discussion}

Despite the significance and prevalence of OIC, there are no clear consensus guidelines for its treatment. One reason for this lack of consensus may be the wide variety of instruments currently available to characterize chronic constipation and the varying array of symptoms that may present in different patients with OIC. Poulsen (2015) makes the point that 'a substantial drawback to using a patients bowel habits for the assessment of OIC is that many patients may have been treated with opioids for a long period of time and in fact may not recall what their normal bowel habits were' [32].

The current consensus for the management of OIC involves a comprehensive step-wise approach. Step one begins with measures designed to prevent the occurrence of OIC and include prophylactic laxatives (stimulant +stool softener and/or an osmotic laxative). Preventative measures should also include lifestyle modifications such as maintaining adequate fluid and fiber intake along with regular exercise. Should OIC still occur in a patient despite the above prophylactic interventions, one should first rule out bowel obstruction and other potential causes of constipation, then titrate doses of laxative/ softener to enhance bowel activity and consider opioid rotation or a dual-action opioid (i.e. tapentadol, tramadol). If constipation persists despite these measures, the provider should then consider prokinetic agents or peripherally-restricted $\mu$-receptor antagonists. Finally, in more extreme cases, one might consider spinal or epidural analgesics or neuroablation to reduce the amount of opioid that is needed.

A potential disconnect may also exist between the OIC that patients are experiencing and their providers awareness of the issue in the patients they are caring for. It is imperative that all health care providers keep an ongoing dialog with their patients so they can be aware of the impact that OIC may be having on their patient's therapeutic compliance, well-being an overall quality of life.

\section{Conclusion}

While opioid analgesics remain the most effective class of agents for the treatment of moderate to severe pain, chronic opioid use is associated with a number of significant adverse effects including constipation. Fortunately, a number of effective therapeutic regimens are available for both treating and preventing opioid-induced constipation. Ongoing research and clinical trials will likely yield additional treatments in the near future to better prevent OIC or provide potent analgesia without significant effects on bowel function.

\section{References}

1. Camilleri M (2011) Opioid-induced constipation: challenges and therapeutic opportunities. Am J Gastroenterol 106: 835-842.

2. Kalso E, Edwards JE, Moore RA, McQuay HJ (2004) Opioids in chronic noncancer pain: systematic review of efficacy and safety. Pain 112: 372-380.

3. Holzer P (2007) Treatment of opioid-induced gut dysfunction. Expert Opin Investig Drugs 16: 181-194.
4. Kurz A, Sessler DI (2003) Opioid-induced bowel dysfunction: pathophysiology and potential new therapies. Drugs 63: 649-671.

5. Wilson L, Knaggs R, Davies E (2010) Opioid-induced bowel dysfunction. Clin Pharm 2: 171-172.

6. Bell TJ, Panchal SJ, Miaskowski C, Bolge SC, Milanova T, et al. (2009) The prevalence, severity, and impact of opioid-induced bowel dysfunction: results of a US and European Patient Survey (PROBE 1). Pain Med 10: 35-42.

7. Bell T, Annunziata K, Leslie JB (2009) Opioid-induced constipation negatively impacts pain management and health-related quality of life: findings from the National Health and Wellness Survey. J Opioid Manag 5: 137-144.

8. Hjalte F, Berggren AC, Bergendahl H, Hjortsberg C (2010) The direct and indirect costs of opioid-induced constipation. J Pain Symptom Manage 40: 696703

9. LoCasale RJ, Datto C, Wilson H, Yeomans K, Coyne KS (2016) The Burden of Opioid-Induced Constipation: Discordance Between Patient and Health Care Provider Reports. J Manag Care Spec Pharm 22: 236-245

10. Williams JT, Ingram SL, Henderson G, Chavkin C, von Zastrow M, et al (2013) Regulation of mu-opioid receptors: desensitization, phosphorylation, internalization, and tolerance. Pharmacol Rev 65: 223-254.

11. Akbarali HI, Inkisar A, Dewey WL (2014) Site and mechanism of morphine tolerance in the gastrointestinal tract. Neurogastroenterol Motil 26: 1361-1367.

12. Dukas L, Willett WC, Giovannucci EL (2003) Association between physical activity, fiber intake, and other lifestyle variables and constipation in a study of women. Am J Gastroenterol 98: 1790-1796.

13. Turan N, AÅ̈̈t TA (2016) The Effect of Abdominal Massage on Constipation and Quality of Life. Gastroenterol Nurs 39: 48-59.

14. Lämås K, Lindholm L, Stenlund $H$, Engström B, Jacobsson $C$ (2009) Effects of abdominal massage in management of constipation--a randomized controlled trial. Int J Nurs Stud 46: 759-767.

15. Kumar L, Barker C, Emmanuel A (2014). Opioid-induced constipation: pathophysiology, clinical consequences, and management. Gastroenterol Res Pract 2014: 6.

16. Tarumi Y, Wilson MP, Szafran O, Spooner GR (2013) Randomized, double-blind placebo-controlled trial of oral docusate in the management of constipation in hospice patients. J Pain Symptom Manage 45: 2-13.

17. Müller-Lissner SA, Kamm MA, Scarpignato C, Wald A (2005) Myths and misconceptions about chronic constipation. Am J Gastroenterol 100: 232-242.

18. Ramkumar D, Rao SS (2005) Efficacy and safety of traditional medical therapies for chronic constipation: systematic review. Am J Gastroenterol 100: 936-971.

19. Pappagallo $M$ (2001) Incidence, prevalence, and management of opioid bowel dysfunction. Am J Surg 182: 11S-18S.

20. Jamal MM, Adams AB, Jansen JP, Webster LR (2015) A randomized, placebocontrolled trial of lubiprostone for opioid-induced constipation in chronic noncancer pain. Am J Gastroenterol 110: 725-732.

21. Cryer B, Katz S, Vallejo R, Popescu A, Ueno R (2014) A randomized study of lubiprostone for opioid-induced constipation in patients with chronic noncance pain. Pain Med 15: 1825-1834.

22. Meissner W, Schmidt U, Hartmann M, Kath R, Reinhart K (2000) Oral naloxone reverses opioid-associated constipation. Pain 84: 105-109.

23. Yuan CS, Foss JF, O'Connor M, Osinski J, Karrison T, et al. (2000) Methylnaltrexone for reversal of constipation due to chronic methadone use: a randomized controlled trial. JAMA 283: 367-372.

24. Yuan CS, Wei G, Foss JF, O'Connor M, Karrison T, et al. (2002). Effects of subcutaneous methylnaltrexone on morphine-induced peripherally mediated side effects: a double blind randomized placebo controlled trial. J Pharmacol Exp Ther 300: 118-123.

25. Leonard J, Baker DE (2015) Naloxegol: treatment for opioid-induced constipation in chronic non-cancer pain. Ann Pharmacother 49: 360-365.

26. Ahmedzai S, Brooks D (1997) Transdermal fentanyl versus sustained-release oral morphine in cancer pain: preference, efficacy, and quality of life. The TTSFentanyl Comparative Trial Group. J Pain Symptom Manage 13: 254-261.

27. Donner B, Zenz M, Strumpf M, Raber M (1998) Long-term treatment of cancer pain with transdermal fentanyl. J Pain Symptom Manage 15: 168-175. 
Citation: Zdanowicz MM (2016) Treatment of Opioid-induced Constipation: A Therapeutic Update. Adv Practice Nurs 2:118. doi:10.4172/25730347.1000118

Page 5 of 5

28. Allan L, Richarz U, Simpson K, Slappendel R (2005) Transdermal fentanyl versus sustained release oral morphine in strong-opioid naïve patients with chronic low back pain. Spine (Phila Pa 1976) 30: 2484-2490.

29. Dale O, Moksnes K, Kaasa S (2011) European Palliative Care Research Collaborative pain guidelines: opioid switching to improve analgesia or reduce side effects. A systematic review. Palliat Med 25: 494-503.

30. Kwong WJ, Hammond G, Upmalis D, Okamoto A, Yang M, et al. (2013) Bowel function after tapentadol and oxycodone immediate release (IR) treatment in patients with low back or osteoarthritis pain. Clin J Pain 29: 664-672.

31. Grond S, Sablotzki A (2004) Clinical pharmacology of tramadol. Clin Pharmacokinet 43: 879-923.

32. Poulsen JL, Brock C, Olesen AE, Nilsson M, Drewes AM (2015) Evolving paradigms in the treatment of opioid-induced bowel dysfunction. Therap Adv Gastroenterol 8: 360-372. 